\title{
Diagnostic Challenge in a Patient Presenting with Ascites and Hypergammaglobulinemia
}

\author{
Syifa Mustika ${ }^{a}$ Mirza Zaka Pratama ${ }^{a}$ Cosmas Rinaldi Adithya Lesmana ${ }^{b}$ \\ ${ }^{a}$ Gastroenterohepatology Division, Department of Internal Medicine, Faculty of Medicine \\ Universitas Brawijaya, Saiful Anwar General Hospital, Malang, Indonesia; ${ }^{b}$ Hepatobiliary \\ Division, Department of Internal Medicine, Faculty of Medicine, Universitas Indonesia, Cipto \\ Mangunkusumo National General Hospital, Jakarta, Indonesia
}

\section{Keywords}

Ascites $\cdot$ Hypergammaglobulinemia $\cdot$ Autoimmune hepatitis

\begin{abstract}
Ascites is defined as the accumulation of intra-peritoneal fluid that can be caused by several diseases. We described a 47-year-old female presenting with low serum-ascites albumin gradient (SAAG) and a markedly high level of serum globulin. Serum protein electrophoresis revealed an $\mathrm{M}$ spike in the gamma region. Other laboratory results showed a marked increase in aspartate aminotransferase and alanine aminotransferase and predominantly conjugated hyperbilirubinemia without a sign of dilatation of bile ducts from abdominal ultrasonography examination. Furthermore, the follow-up showed a positive result for the anti-nuclear antibody test. The patient was assessed with autoimmune hepatitis, and the cause of ascites was suggested from portal hypertension although the level of SAAG was low. The ascites condition got improved after salt restriction, diuretics treatment, and abdominal paracentesis. However, the patient passed away because of the intracranial hemorrhage as a result of prolonged INR and APTT due to liver failure.
\end{abstract}

\section{Introduction}

Ascites is defined as the excess accumulation of intra-peritoneal fluid which can be an important clue to a significant underlying illness. Ascites is broadly classified into transudate and exudate based on the protein content with a potentially wide range of differential diagnosis. 
Liver cirrhosis (LC) is the most common underlying disease that can cause ascites [1]. Serumascites albumin gradient (SAAG) is widely used to differentiate the cirrhotic or noncirrhotic ascites with a sensitivity of $90 \%$ and a specificity of $77.5 \%$ [2]. SAAG has been also documented to be more superior in differentiating the possible cause of ascites when compared to ascitic fluid total protein, ascitic fluid cholesterol, or serum-ascites cholesterol gradient [3]. However, in some conditions, SAAG can be falsely low in a patient with LC, and therefore the diagnosis can be difficult [4].

On the other hand, gamma globulin is a class of globulin that can be increased in several diseases, such as multiple myeloma, amyloidosis, malignant lymphoma, Waldenström's macroglobulinemia, and chronic infections [5]. Hypergammaglobulinemia also can be present in patients with chronic liver disease due to the diminished removal of immunoglobulins by the diseased liver [6]. Hypergammaglobulinemia with the presence of autoantibodies and the exclusion of viral hepatitis infection or other toxins which may cause the liver injury can be also caused by autoimmune hepatitis (AIH). AIH is a rare chronic liver disease with the prevalence estimates from 4 to 42.9 cases per 100,000 persons with reported annual incidences ranging from 0.67 to 2.23 cases per 100,000 persons [7]. However, serum protein electrophoresis is not a routine examination in patients with ascites. Therefore, we would like to report a case of 47-year-old female with the presentation of ascites, a low gradient of SAAG, and hypergammaglobulinemia.

\section{Case Report}

A 47-year-old female was referred to the hospital due to gradual abdominal enlargement accompanied by leg swelling lasting for 2 weeks. There was also a decrease in body weight about $10 \mathrm{~kg}$ in the last 8 months. The starting weight of the patient was $67 \mathrm{~kg}$ and reduced to $57 \mathrm{~kg}$ in the past 8 months. General weakness, pruritus, and decreased pubic and axillary hair were also noticeable. Eight months earlier, she had profound jaundice without abdominal pain. At that time, she got ursodeoxycholic acid and got better. After 2 months of treatment, the patient was lost to follow-up and never visited again to the hospital. She had no noteworthy medical or family history, including gastrointestinal bleeding or liver disease. On physical examination, the patient was alert, fully oriented, and the vital sign was stable. There were pale conjunctiva and icteric of the sclera. Her abdomen was round and soft with severe ascites. There was no abdominal tenderness. Mass or organomegaly was difficult to assess due to massive ascites. Bilateral pitting edema was present on both ankles.

Initial laboratory data are shown in Table 1; it revealed that she had macrocytic anemia, thrombocytopenia, and leukocytosis. The liver function test showed an increase in bilirubin level with mainly direct hyperbilirubinemia with an increase in AST and ALT with AST/ALT ratio $>1$. ALP and $\gamma$-GT levels were normal. Hemostatic functions showed an increase in both INR and APTT. Albumin levels were decreased with an increase in globulin levels. Renal function tests were still normal, and the results of hepatitis B and C were negative. Compared to the laboratory results from 8 months ago, the AST and ALT levels were higher and also the levels of bilirubin more severe compared to the present admission. However, there was no anemia nor thrombocytopenia documented 8 months ago. Ascites fluid analysis showed that the fluid was clear and yellow colored with low levels of protein, LDH, leukocyte, and erythrocyte. SAAG was $<1.1 \mathrm{mg} / \mathrm{dL}$. Cytology of ascetic fluid analysis found some polymorphonuclear and mononuclear cells without malignant cells. The results of ascites fluid analysis are also shown in Table 1.

The urinalysis result showed that there was moderate bilirubinuria, but no proteinuria was found. We also found mild urobilinogen in the urine. We already excluded the cause of hypoalbuminemia was due to nephrotic syndrome because no nephrotic-range proteinuria was found based on the examination of protein-creatinine ratio or albumin-creatinine ratio.

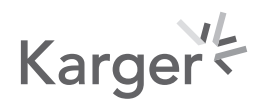


Table 1. Laboratory results

\begin{tabular}{|c|c|c|c|}
\hline Variables & Results (8 months before) & Results (this admission) & Normal value \\
\hline \multicolumn{4}{|l|}{ Complete blood count } \\
\hline Hemoglobin, g/dL & 11.7 & 6.3 & $11.4-15.1$ \\
\hline Leukocyte, / $\mu \mathrm{L}$ & 13,930 & 18,990 & $4,700-11,300$ \\
\hline Hematocrit, \% & 33.1 & 18.3 & $38-42$ \\
\hline Thrombocytes, / $\mu \mathrm{L}$ & 286,000 & 126,000 & $142,000-424,000$ \\
\hline MCV, fL & 85.1 & 93.5 & $80-93$ \\
\hline MCH, pg & 30.1 & 31.5 & $27-31$ \\
\hline \multicolumn{4}{|l|}{ Differential count, \% } \\
\hline Eosinophil & 7.3 & 3.3 & $0-4$ \\
\hline Basophil & 1.6 & 0.2 & $0-1$ \\
\hline Neutrophil & 49.9 & 64.4 & $51-67$ \\
\hline Lymphocyte & 30.9 & 22.3 & $25-33$ \\
\hline Monocyte & 10.3 & 9.8 & $2-5$ \\
\hline \multicolumn{4}{|l|}{ Hemostatic function } \\
\hline PPT, s & No data & 32.6 & $9.4-11.3$ \\
\hline APTT, s & No data & 68.9 & $24.6-30.6$ \\
\hline INR & No data & 3.43 & $<1.5$ \\
\hline \multicolumn{4}{|l|}{ Liver function test } \\
\hline AST, U/L & 656 & 166 & $0-32$ \\
\hline ALT, U/L & 265 & 78 & $0-33$ \\
\hline Total bilirubin, mg/dL & 8.77 & 4.33 & $<1.0$ \\
\hline Direct bilirubin, mg/dL & 8.55 & 3.43 & $<0.25$ \\
\hline Indirect bilirubin, $\mathrm{mg} / \mathrm{dL}$ & 0.22 & 0.90 & $<0.75$ \\
\hline ALP, U/L & 161 & 129 & $66-220$ \\
\hline$\gamma$-GT, U/L & 26 & 23 & $5-36$ \\
\hline Albumin, gr/dL & No data & 1.27 & $3.5-5.5$ \\
\hline Globulin, gr/dL & No data & 7.08 & $2.5-3.5$ \\
\hline \multicolumn{4}{|l|}{ Renal function test } \\
\hline Urea, mg/dL & 32.3 & 43.4 & $16.6-48.5$ \\
\hline Creatinine, mg/dL & 0.84 & 1.25 & $<1.2$ \\
\hline Calcium, mg/dL & No data & 8.4 & $7.6-11.0$ \\
\hline \multicolumn{4}{|l|}{ Hepatitis viral markers } \\
\hline HbsAg & No data & Nonreactive & \\
\hline Anti-HCV & No data & Nonreactive & \\
\hline \multicolumn{4}{|l|}{ Autoimmune markers } \\
\hline ANA test (ELISA) & No data & 2.4 & $<1$ ratio \\
\hline \multicolumn{4}{|l|}{ Ascites fluid analysis } \\
\hline \multicolumn{4}{|l|}{ Macroscopic analysis } \\
\hline Color & No data & Yellow & \\
\hline Clot & No data & Negative & \\
\hline Clarity & No data & Clear & \\
\hline
\end{tabular}


Mustika et al.: A Patient with Ascites and Hypergammaglobulinemia

Table 1 (continued)

\begin{tabular}{|c|c|c|c|}
\hline Variables & Results (8 months before) & Results (this admission) & Normal value \\
\hline \multicolumn{4}{|l|}{ Microscopic analysis } \\
\hline Erythrocyte count, $/ \mu \mathrm{L}$ & No data & 100 & \\
\hline Leukocyte count, / $\mu \mathrm{L}$ & No data & 120 & \\
\hline PMN, \% & No data & 10 & \\
\hline MN, \% & No data & 90 & \\
\hline \multicolumn{4}{|l|}{ Chemical analysis } \\
\hline Protein, gr/dL & No data & 0.9 & \\
\hline Glucose, mg/dL & No data & 93 & \\
\hline Triglyceride, mg/dL & No data & 13 & \\
\hline Cholesterol, mg/dL & No data & 7 & \\
\hline $\mathrm{LDH}, \mathrm{U} / \mathrm{L}$ & No data & 100 & \\
\hline Albumin, mg/dL & No data & 0.46 & \\
\hline SAAG & No data & 0.81 & \\
\hline Cytological analysis & \multicolumn{3}{|c|}{ No malignant cell was found; background: PMN and MN cells } \\
\hline
\end{tabular}

PMN, polymorphonuclear; MN, mononuclear; AST, aspartate aminotransferase; ALT, alanine aminotransferase; SAAG, serum-ascites albumin gradient.

Compared to the results from 8 months ago, the bilirubin and urobilinogen in the urine were milder in this admission with the other results similar. The urinalysis results are shown in Table 2.

Abdominal ultrasound examination (shown in Fig. 1) showed ascites fluid in perivesical, perihepatic, perisplenic, hepatorenal fossa, and peri-intestine regions. No mass or organomegaly was found. Previous abdominal ultrasound from 8 months ago showed no ascites, but there was a thickening of the wall of the gall bladder without a sign of biliary obstruction. Because of the high level of globulin in this patient, we performed plasma protein electrophoresis in this patient. It showed a monoclonal gammopathy with an $\mathrm{M}$ spike in the gamma region with an increased level of $\gamma$-globulin. The result of plasma protein electrophoresis examination is shown in Figure 2.

While it is unfortunate that there was a lack of data regarding the bone marrow analysis in this patient, we found no sign of organ damage related to suspicion of multiple myeloma regarding the hypergammaglobulinemia in this patient. There were no lytic lesion from bone survey, hypercalcemia, or renal dysfunction from this patient. Because we suspected the possibility of autoimmunity in this patient, we found that there was an increased level of serum anti-nuclear antibody (ANA) by ELISA.

Based on the current data, the ascites was thought due to portal hypertension that might be caused by AIH. The patient was restricted for sodium intake and was given spironolactone $100 \mathrm{mg}$ QD, furosemide $40 \mathrm{mg}$ QD, ursodeoxycholic acid $250 \mathrm{mg}$ b.i.d., and prednisone $60 \mathrm{mg} /$ day. She also underwent large-volume paracentesis for the ascites followed by albumin transfusion. We also administered vitamin K injection and transfusion of fresh frozen plasma to correct the hemostasis function; however, there was no improvement regarding prolongation of INR and APTT.

Clinical improvements were found for the ascites and the laboratory results after 1-week treatment. She visited the Gastroenterohepatology outpatient clinic 1 week later, and there was a clinical improvement for the ascites showed by the decrease in abdominal circumference with a decrease in body weight of $2 \mathrm{~kg}$ in 1 week. The monitoring of laboratory results is shown in Figure 3.

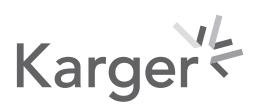


Mustika et al.: A Patient with Ascites and Hypergammaglobulinemia

Table 2. Urinalysis results

\begin{tabular}{llll}
\hline Variables & Results (8 months before) & Results (this admission) & Normal value \\
\hline $\begin{array}{l}\text { Macroscopic analysis } \\
\text { Clarity }\end{array}$ & Clear & Slight cloudy & \\
Color & Yellow & Yellow & \\
Microscopic analysis & & & \\
Epithelia, /LPF & 7.0 & 7.8 & $<3$ \\
Erythrocyte, /HPF & 1.6 & 5.5 & $<3$ \\
Leukocyte, /HPF & 5.7 & 9.4 & $<5$ \\
Crystal, /HPF & Negative & Negative & Negative \\
Chemical analysis & & & \\
pH & 6.0 & 6.0 & $4.5-8.0$ \\
Specific gravity & 1,025 & 1,020 & $1,005-1,030$ \\
Glucose & Negative & Negative & Negative \\
Protein & Negative & Negative & Negative \\
Protein-creatinine ratio, $\mathrm{mg} / \mathrm{gCr}$ & - & 200 & \\
Albumin-creatinine ratio, $\mathrm{mg} / \mathrm{gCr}$ & - & 10 & \\
Ketone & Negative & Negative & Negative \\
Bilirubin & +3 & +2 & Negative \\
Urobilinogen, $\mu$ mol/L & 33 & 16 & $<17$ \\
Nitrite & Negative & Negative & Negative \\
Leukocyte & +1 & Trace & Negative \\
Blood & Negative & Negative & Negative \\
\hline
\end{tabular}

The patient was planned to do magnetic resonance cholangiopancreatography and liver biopsy afterward. However, 1 week later, she visited the outpatient Gastroenterohepatology clinic. She had a sudden decrease of consciousness in the morning with the suspicion of stroke with intracranial hemorrhage. She died because of brain herniation due to the intracranial hemorrhage.

\section{Discussion}

According to the data based on the case presentation, we assessed this patient with transudative ascites due to portal hypertension. The most useful examination to distinguish the exudate and transudate ascites was the SAAG examination. SAAG had a sensitivity of $90 \%$ and a specificity of $77.5 \%$ in differentiating cirrhotic from noncirrhotic ascites at the cutoff value of $>1.1 \mathrm{gr} / \mathrm{dL}[1,5]$. Noncirrhotic ascites with SAAG levels $<1.1 \mathrm{gr} / \mathrm{dL}$ is usually accompanied by a high level of albumin, protein, LDH, and cholesterol from the ascites. However, there were some conflicting data from the result of ascites fluid examination, although we found that low gradient of SAAG, total protein, LDH, and cholesterol were also low in this patient. Low SAAG and low total protein from the ascites are usually found in the nephrotic syndrome or protein-losing enteropathy. However, there was no nephrotic-range proteinuria nor sign of gastrointestinal disease in this patient. Patients with protein-losing enteropathy usually have decreased level of both albumin and globulin with a low level of gamma globulin; however, in this patient, we found 

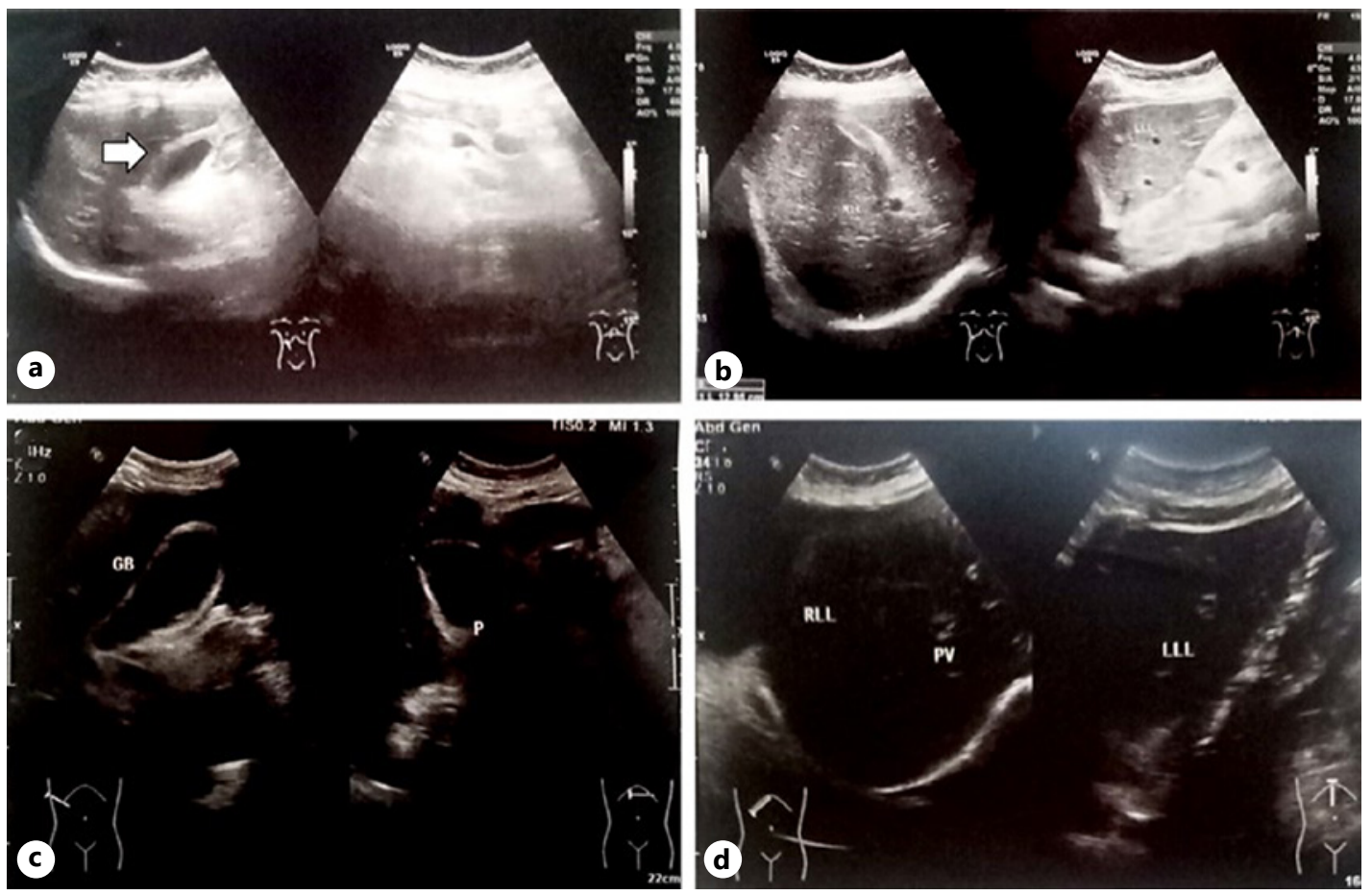

Fig. 1. Abdominal USG examination. A, B Abdominal USG from 8 months ago. There was a thickening of the wall of the gall bladder (shown by a marker). C, D Abdominal USG from this admission. No abnormality was found from the liver and hepatobiliary tract.

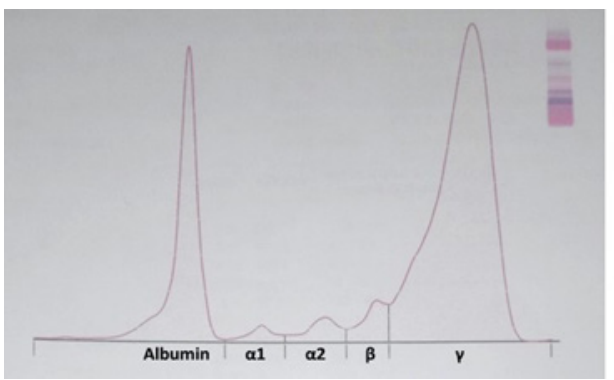

\begin{tabular}{lcccc}
\hline Fractions & $\%$ & $\begin{array}{c}\text { Normal Value } \\
(\%)\end{array}$ & $\begin{array}{c}\text { Concentration } \\
(\mathrm{mg} / \mathrm{dl})\end{array}$ & $\begin{array}{c}\text { Normal Value } \\
(\mathrm{mg} / \mathrm{dl})\end{array}$ \\
\hline Albumin & 24.6 & $55.8-66.1$ & 1.68 & $4.02-4.76$ \\
Alpha-1 & 1.8 & $2.9-4.9$ & 0.12 & $0.21-0.35$ \\
Alpha-2 & 3.2 & $7.1-11.8$ & 0.22 & $0.51-0.85$ \\
Beta & 4.1 & $8.4-13.1$ & 0.28 & $0.60-9.40$ \\
Gamma & 66.3 & $11.1-18.8$ & 4.53 & $0.80-1.35$ \\
\hline
\end{tabular}

Fig. 2. Result of plasma protein electrophoresis examination.

a high level of gamma globulin [8]. Therefore, despite the low gradient of SAAG, we still assessed this patient due to portal hypertension. Sastry et al. [3] also revealed that some conditions might cause the falsely low value of SAAG usually when the albumin serum level was low. Although quite uncommon, some cases of portal hypertension might also have a low gradient of SAAG [9].

We also suspected that portal hypertension found in this patient was part of the sign of LC. There were other features of LC found in this patient, such as jaundice, loss of pubic and axillary hair, prolonged hemostatic function (PPT and APTT), hypoalbuminemia, macrocytic anemia, and thrombocytopenia, although splenomegaly was not prevalent in this patient. The APRI and FIB-4 scores were 2.6 and 5.45, respectively, which indicated severe fibrosis. Regardless of the cause of the LC from this patient, the ascites improved after the initial treatment with salt restriction and diuretics. Most guidelines recommend that dietary restriction and diuretics, such as spironolactone and furosemide, are still the mainstays of therapy of ascites due to cirrhosis [10]. 


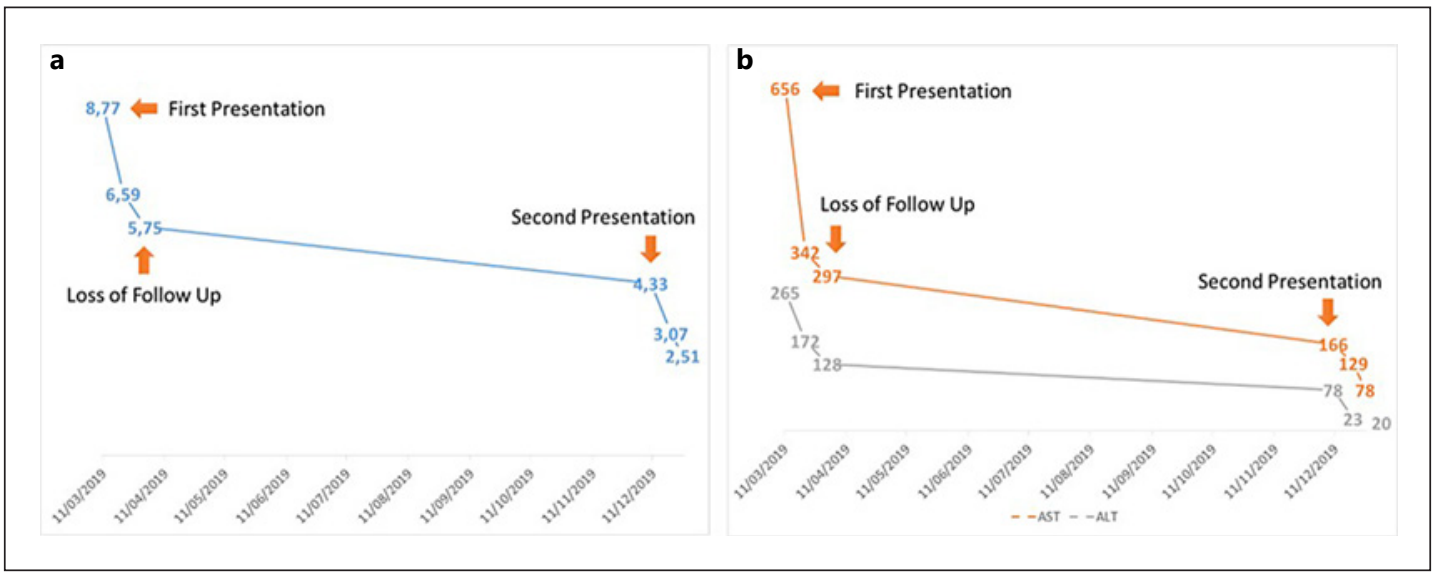

Fig. 3. Monitoring of liver function tests. A Total bilirubin and B AST and ALT from the first presentation until second presentation. AST, aspartate aminotransferase; ALT, alanine aminotransferase.

The diagnosis of AIH is based on the criteria that were established and modified by the international AIH group, which include predominant elevation in the levels of serum aminotransferases, negative markers for viral hepatitis, exclusion of genetic liver diseases, absence of toxic or alcohol liver injury, presence of autoantibodies for AIH, elevated levels of serum IgG, and histological features of AIH [11]. The presence of the ANA besides the hepatocellular injury and intrahepatic cholestasis with increased levels of gamma globulin in this patient were highly suspicious for AIH. Although there was a lack of data of the autoantibody profiles of AIH markers and liver biopsy, a patient with ANA and/or anti-smooth muscle antibody was classified with type 1 or classic AIH. Moreover, there was no sign of hepatitis viral infections and absence of toxic or alcohol liver injury from this patient. AIH type 1 was the most common type of AIH and mostly found in females than males [12]. Untreated patients with AIH could further develop LC. The prognosis and survival of the patients with AIH are usually good because they respond well to corticosteroid and immunosuppressant therapy [13]. Although we found some improvement in the clinical and laboratory data of this patient, this patient passed away due to the complication of coagulopathy which caused intracranial bleeding.

According to the serum protein electrophoresis examination, we found that the patient also had hypergammaglobulinemia. Gamma globulin in conventional protein electrophoresis consists of 5 immunoglobulins, of which $80 \%$ was immunoglobulin G (IgG) [5]. Hypergammaglobulinemia is usually seen in patients with chronic liver disease [6]. On the other hand, an elevated serum of gamma globulin or IgG is considered as the best diagnostic marker for AIH. According to the guideline of diagnosis and management of AIH from the American Association of the Study of Liver Disease (AASLD), the elevation of gamma globulin or IgG level $>1.5$ times the upper normal limit is definite for the diagnosis of AIH [12].

$\mathrm{AIH}$ is considered as a rare condition that causes LC in the Indonesian population. Therefore, AIH is usually underdiagnosed. It was quite unfortunate that this patient has been missed diagnosis and management for AIH at the first admission. Most patients with AIH have a good prognosis after the administrations of corticosteroids and immunosuppressants. However, the survival of AIH patients with LC at the time of diagnosis is significantly lower compared to noncirrhotic patients [13]. Regarding the cause of death in this patient, the bleeding risk has been known to be increased in a patient with cirrhosis. Abnormality of hemostatic profile and thrombocytopenia are the most common predisposing factors of bleeding in a patient with cirrhosis. As been reported before, the risk of spontaneous intracranial hemorrhage is increased in cirrhosis patients [14]. In another cohort study, the hazard 
ratio of intracranial hemorrhage in the patients with cirrhosis was 1.62 (95\% CI: 0.85-3.10) when compared to noncirrhotic patients [15].

In conclusion, not all patients with low SAAG have noncirrhotic ascites, and therefore a careful examination of the ascites fluid analysis is a vital part to determine the proper diagnosis and management in the patient. Most AIH patients have a good prognosis after receiving immunosuppressant therapy. However, the survival of the AIH patients with cirrhosis is lower when compared to noncirrhotic patients.

\section{Acknowledgments}

The authors thank the resident in charge who helped monitoring of the patient in the ward and polyclinics. The authors also thank the radiology department who helped with abdominal ultrasonography examination of the patient.

\section{Statement of Ethics}

The authors have no ethical conflicts to disclose. The study was performed following the ethical standards lain down in the Declaration of Helsinki and was approved by the Medical Ethics Committee of Human Research of Saiful Anwar General Hospital Malang, Indonesia. The ethics committee did not provide an approval reference number; however, the study was approved on December 19, 2019. Written informed consent was obtained from the patient's husband for publication of this case report and any accompanying images.

\section{Conflict of Interest Statement}

The authors declare that there are no conflicts of interest to disclose.

\section{Funding Sources}

There was no funding source for this report.

\section{Author Contributions}

All authors wrote and edited the manuscript. Syifa Mustika contributed to conception, design, analysis, interpreting the data, drafting, revisiting the article, and final approval. Mirza Zaka Pratama contributed to conception, design, analysis, interpreting the data, drafting, revisiting the article, and final approval. Cosmas Rinaldi Adithya Lesmana contributed to design, analysis, drafting, revisiting the article, and final approval.

\section{Data Availability Statement}

All data generated or analyzed during this study are included in this article. Further enquiries can be directed to the corresponding author. 
Mustika et al.: A Patient with Ascites and Hypergammaglobulinemia

\section{References}

1 Rudralingam V, Footitt C, Layton B. Ascites matters. Ultrasound. 2017;25(2):69-79.

2 Prabhu M, Gangula RS, Stanley W. Diagnostic utility of serum ascites lipid and protein gradients in differentiation of ascites. Int J Hepatol. 2019;2019:8546010.

3 Sastry AS, Mahapatra SC, Dumpula V. Ascitic fluid analysis with special reference to serum ascites cholesterol gradient and serum ascites albumin gradient. Int J Res Med Sci. 2017;5(2):429.

4 Seth AK, Rangarao R, Pakhetra R, Baskaran V, Rana P, Rajamani S. Accuracy of serum - ascites albumin gradient in the aetiological diagnosis of ascites. Med J Armed Forces India. 2002;58(2):124-6.

5 Busher JT. Serum albumin and globulin. In: Walker HK, Hall WD, Hurst JW, editors. Clinical methods: the history, physical, and laboratory examinations. 3rd ed. Boston: Butterworths; 1990.

6 Cacciola I, Filomia R, Alibrandi A, Franzè MS, Caccamo G, Maimone S, et al. Hypergammaglobulinemia is a strong predictor of disease progression, hepatocellular carcinoma, and death in patients with compensated cirrhosis. Liver Int. 2018;38(7):1220-9.

7 Kim BH, Choi HY, Ki M, Kim KA, Jang ES, Jeong SH. Population-based prevalence, incidence, and disease burden of autoimmune hepatitis in South Korea. PLoS One. 2017 Aug 3;12(8):e0182391.

8 Levitt D, Levitt M. Protein losing enteropathy: comprehensive review of the mechanistic association with clinical and subclinical disease states. Clin Exp Gastroenterol. 2017;10:147-68.

9 Khandwalla HE, Fasakin Y, El-Serag HB. The utility of evaluating low serum albumin gradient ascites in patients with cirrhosis. Am J Gastroenterol. 2009;104(6):1401-5.

10 Runyon BA. Introduction to the revised American association for the study of liver diseases practice guideline management of adult patients with ascites due to cirrhosis 2012. Hepatology. 2013;57(4):1651-3.

11 Bogdanos DP, Invernizzi P, Mackay IR, Vergani D. Autoimmune liver serology: current diagnostic and clinical challenges. World J Gastroenterol. 2008;14:3374-87.

12 Manns MP, Czaja AJ, Gorham JD, Krawitt EL, Mieli-Vergani G, Vergani D, et al. Diagnosis and management of autoimmune hepatitis. Hepatology. 2010;51(6):2193-213.

13 Gleeson D. Long-term outcomes of autoimmune hepatitis. Clin Liver Dis. 2019;14(1):24-8.

14 Parikh NS, Navi BB, Kumar S, Kamel H. Association between liver disease and intracranial hemorrhage. J Stroke Cerebrovasc Dis. 2016 Mar 1;25(3):543-8.

15 Lai CH, Cheng PY, Chen YY. Liver cirrhosis and risk of intracerebral hemorrhage: a 9-year follow-up study. Stroke. 2011;42(9):2615-7. 